Research Article

\title{
Big NB-IoT Data: Enhancing Portability of Handheld Narrow-Band Internet of Things Performance on Big Data Technology
}

\author{
Jie $\mathrm{Xu}$ (iD \\ Anhui Technical College of Industry and Economy, Hefei 230051, China \\ Correspondence should be addressed to Jie Xu; xujie@ahiec.edu.cn
}

Received 28 May 2021; Revised 18 June 2021; Accepted 3 July 2021; Published 12 July 2021

Academic Editor: Fazlullah Khan

Copyright (c) 2021 Jie Xu. This is an open access article distributed under the Creative Commons Attribution License, which permits unrestricted use, distribution, and reproduction in any medium, provided the original work is properly cited.

\begin{abstract}
The Narrow Band-Internet of Things (NB-IoT) is a wideband radio technology developed for the Internet of Things that enables smoother- and farther-reaching connectivity between IoT devices. In addition to traditional network optimization devices, Bluetooth and $\mathrm{Wi}-\mathrm{Fi}$, its virtue is low cost, and it consumes less energy and has high coverage and extended battery life. In order to secure the balance of task execution latency across NB-IoT devices, in this research work, we design a handheld NB-IoT wireless communication device. Furthermore, we provide realistic resource-sharing methods between multimedia and sensor data in NBIoT wireless deployment by our accurate analytical methodology. In addition, we have considerably enhanced technology for gathering Big Data from several scattered sources, in combination with advancements in big data processing methodologies. The proposed handheld terminal has a wide variety of commercial applications in intelligent manufacturing and smart parking. Simulation outcomes illustrate the benefits of our handheld terminal, which provides practical solutions for network optimization, improving market share and penetration rate.
\end{abstract}

\section{Introduction}

The Internet of Things is a new technology in the world of telecommunications. The Internet of Things (IoT) is a network of physical items or things integrated with software, electronics, sensors, and connections, allowing them to attain better value and service by communicating information with suppliers, operators, and other smart devices [1]. Various IoT-based applications have emerged in recent years due to the fast growth of IoT technology in the domestic and industry. The overall revenue of the domestic IoT industry [2,3] has exceeded 30 trillion yuan in 2018-2020. These include smart city, smart metering, health monitoring, automatic driving, smart manufacturing, and many others [4]. The proportion of IoT technology used in the industry also continues to increase every year. It has entered many sectors, including application in manufacturing [5], transportation and logistics [6], health care $[7,8]$, consumer electronics [9], retail [10, 11], and automobile [12]. Mainly, the data collection and processing unit producing new captors is responsible for the study of the IoT-enabled gadgets. However, it generally does not affect the network that integrates IoT devices into the Internet simply by using current computer network solutions such as WIFI or Bluetooth. These computer networking devices have not even been meant to be small computers for low-power devices such as remote sensors [13].

Narrowband IoT (NB-IoT) based on a cellular network has become a new communication option with several advantages for the Internet of Things. For instance, it is covered by a huge and profound $20 \mathrm{~dB}+$, a small power consumption, a cheap cost, and wide connection, i.e., $50 \mathrm{~K}$ user capacity/200 KHz cell [14]. NB-IoT technology is in full swing in the overall area of network development, and demand for network optimization will continue to increase. The present network improvements for NB-IoT are based on a PC page of + ATU. ATU is a device of a schoolbag size. ATU completes the acquisition and completion of the ATU 
collection of the reporting NB-IoT data analyses and associated duties of the NB-IoT network signal network terminal. In practice, there are several problems for the present NBIoT network optimization equipment. Firstly, poor portability is required, i.e., network optimization, which is uncomfortable to use for testing using the PC + ATU. Second, short test time, i.e., PC + ATU, is driven by inbuilt inverters, which are quite powerful. Moreover, several special resource management-related issues are posed by collecting such heterogeneous big data, where reliable data streams play a significant role [15].

In this research, we aim to overcome this problem by providing an analytical framework for predicting the performance of the heterogeneous gathering of data across a handheld NB-IoT terminal. We have developed a handheld NB-IoT-based information management solution for traditional devices to solve the existing challenges of device administration. The proposed handheld NB-IoT enhanced management efficiency by collecting information, enabling real-time perception, accurate placement, and periodic information gathering and updating to identify each control of devices uniquely. Using the transparent CDMA baseband decoding capabilities, the suggested system may perform active performance testing while concurrently obtaining user and signal data. We subsequently used this tool to investigate the everyday operations of a handheld NB-IoT network from the standpoint of site verification and network maintenance.

The rest of the paper is organized as follows: In Section 2, a proposed system model design of the NB-IoT system is outlined. The communication process analysis is conducted in Section 3. The structure design of NB-IoT wireless communication equipment is further summarized in Section 4. The structure and big data evaluation are discussed in Sections 5 and 6 , concluding the paper with summary and future research directions.

\section{Proposed System Design}

This section introduces the proposed system design, i.e., handheld NB-IoT wireless communication terminal device, to address flaws in present technologies. Through research on the device, terminal, and medium, it can emphasize its mobility, small size, low power consumption, and complete the optimization of the NB-IoT network by utilizing the handheld terminal. The system design framework is shown in Figure 1.

Handheld NB-IoT wireless communication equipment includes an NB-IoT antenna to collect NB-IoT wireless signals and transmit them to the NB-IoT communication module [16]. The NB-IoT communication module receives the NB-IoT wireless signal. It conducts demodulation, downconversion, signaling reading, and network optimization on the NB-IoT wireless signal to obtain the processing data. It sends the processing data to the client. The communication driver module of the client realizes the communication between the client and the NB-IoT communication module [17]. The client TCP/IP protocol stack module receives and processes the data and maps it into the network card device, so that the handheld terminal can recognize and realize the network transmission of data. The device block diagram of the handheld NB-IoT network optimization system is shown in Figure 2. In Figure 2, 1 refers to the NB-IoT wireless communication device, and 2 refers to the handheld terminal.

Traditional PC + ATU devices are built on Windows, and peripheral ATU devices provide acquisition signaling data to the PC over the USB interface. Each ATU module requires installing the matching driver and Windows application program on the Windows system, which is hard to use. The peripheral driver is not available for the Android system, which runs on portable terminals. Due to the operating system's security protection, the operating system cannot finish the interaction with the external user's USB devices when the handheld terminal communicates and interacts with the peripheral user-defined USB devices. As a result, we isolate the USB Core driver for the operating system and write it to the client directly. The application software is used to finish the installation and registration of the USB Core driver, allowing data connection between the portable terminal and our NB-IoT communication module $[18,19]$.

The data is received and processed by the client TCP/IP protocol stack module. It maps it into a network card device that the portable terminal recognizes and enables data transmission over the network. To be more explicit, the typical PC + ATU needs to map the NB-IoT module into a network card device because of Windows' open interface. The Windows system can recognize and transfer data using the TCP/IP stack associated with the Windows system [20]. However, due to handset operating system security protection, special USB device drivers cannot register directly to the handset operating system. NB-IoT communication module cannot provide a normal mapping into the operating system. To identify the network adapter device, the operating system with TCP/IP protocol stack cannot be used. Therefore, handheld terminals in the client used TCP/ IP protocol stack [21-23].

\section{Communication Process Analysis}

The communication standard for NB-IoT is intended to enable IoT devices to function over carrier networks in an underused "guard band" between LTE or independently of an existing Global System for Mobile (GSM) communication carrier wave. NB-IoT communication analysis performs the following several steps of the process. Firstly, the NB-IoT antenna collects NB-IoT wireless signals and transmits them to the NB-IoT communication module [24]. The NB-IoT communication module receives the NB-IoT wireless signal. It conducts demodulation, downconversion, signaling reading, and network optimization on the NB-IoT wireless signal. NB-IoT obtains the processing data and sends the processing data to the client through the data interface. Secondly, the data interface is arranged at the bottom of the back cover. The back cover can be disassembled and installed on the back of the handheld terminal through its data interface plugged into the data interface of the handheld terminal. Thirdly, the back cover is also provided with a 


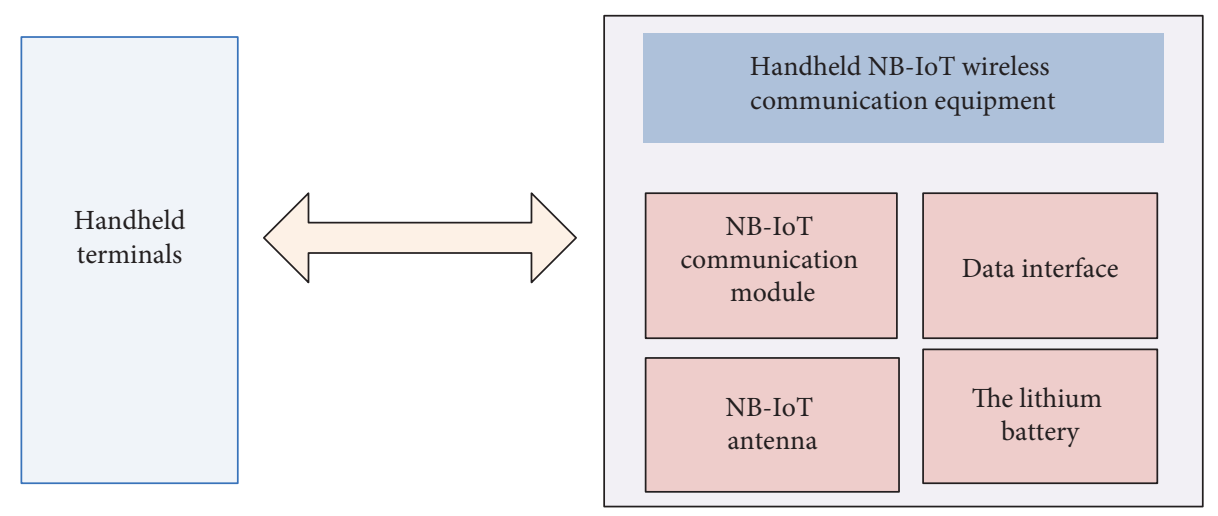

FIgURE 1: Fine-grained access control model.

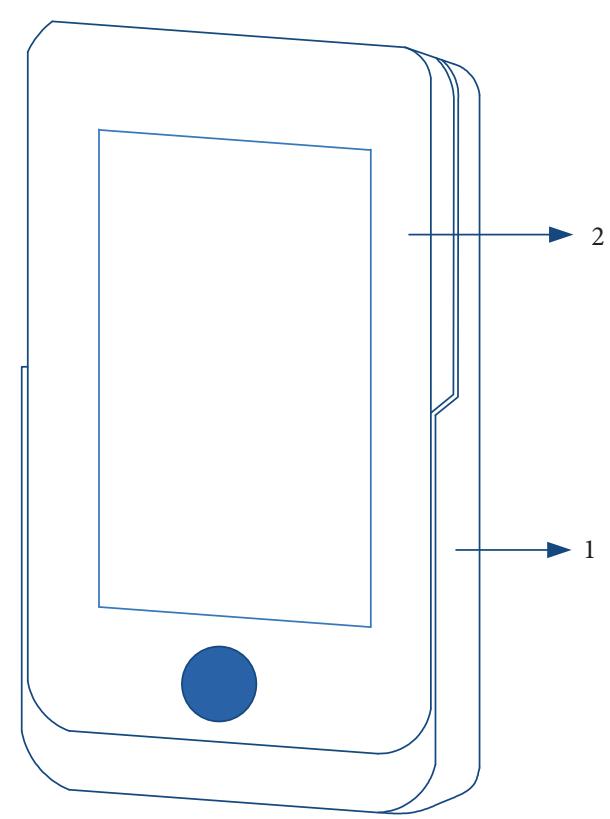

FIGURE 2: Handheld terminal NB-IOT wireless communication module.

lithium battery, electrically connected with the NB-IoT communication module, NB-IoT antenna, and data interface. Finally, a charging port is arranged near the position of the lithium battery on the back cover, and a charging circuit is arranged on the back cover. The charging port is electrically connected with the input end of the charging circuit, and the output end of the charging circuit is electrically connected with the lithium battery.

\section{Structure Design of NB-IoT Wireless Communication Equipment}

In this section, the structure of your NB-IoT terminal frequently references LTE-M for context, in part because the NB-IoT architecture is quite similar to the LTE-M design. NB-IoT requires a lower bandwidth channel $(200 \mathrm{kHz}$ vs. $1.4 \mathrm{MHz}$ ), and devices can operate in nontraditional bands such as LTE security channels. The schematic diagram of the
NB-IoT wireless communication device installed on the handheld terminal is shown in Figure 3. Handheld NB-IoT wireless communication equipment is added on the rear cover 1. NB-IoT communication module 11, NB-IoT antenna 12 , and data interface 13 are installed on the rear cover $[13,25]$. NB-IoT antenna 12 is used to collect NB-IoT wireless signals and transmit them to NB-IoT communication module 11 . The NB-IoT Communication Module 11 receives the NB-IoT wireless signal. It conducts demodulation, downconversion, signaling reading, and network optimization on the NB-IoT wireless signal to obtain the processing data and sends the processing data to the client through the data interface; the data interface 13 is arranged at the bottom of the back cover 1 , and the back cover 1 can be disassembled and installed on the back of the handheld terminal 2 by plugging the data interface 13 into the data interface of the handheld terminal 2.

The handheld NB-IoT wireless communication device is installed on a handheld terminal through a data interface when used. The NB-IoT network optimization test is realized by combining and matching the wireless communication module of the handheld terminal NB-IoT. The data interface of the handheld terminal (USB interface or Type C interface) is connected with the NB-IoT communication module. NBIoT communication module completes the acquisition of NB-IoT network signals [26]. Handheld terminal is used to complete NB-IoT wireless communication module data analysis and related tasks. The rear cover is also provided with lithium battery 14 , electrically connected with NB-IoT communication module 11 , NB-IoT antenna 12, and data interface 13. Lithium-ion batteries power electronic devices. The rear cover 1 is provided with a charging port 15 near the lithium battery 14, and the rear cover 1 is also provided with a charging circuit. The charging port is electrically connected with the input end of the charging circuit, and the output end of the charging circuit is electrically connected with the lithium battery. The handheld NB-IoT wireless communication device can be directly charged to the lithium battery. The rear cover includes a backplate; the bottom edge of the backplate is provided with a base 16 , and the base 16 is provided with a mounting surface perpendicular to the backplate. The data interface is vertically arranged on the mounting surface of the backplate [20]. 


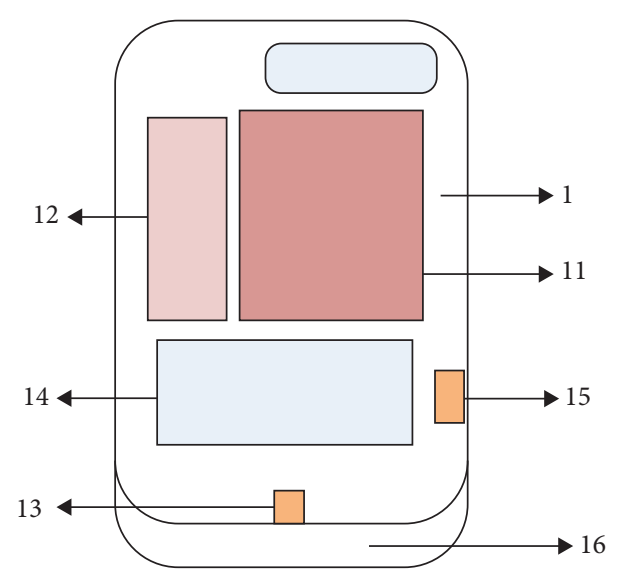

Figure 3: Schematic diagram of the NB-IoT wireless communication device.

The back of the back cover is in the same shape as the back of the handheld terminal. The back cover is closed on the back of the handheld terminal in that it is plugged into the data interface of the handheld terminal through its data interface. The back cover is consistent with the shape of the handheld terminal. After the back cover is installed, the back cover is consistent with the shape of the shell of the mobile phone terminal. The bottom of the back cover is provided with a base. USB is arranged on the base, so that after the back cover is installed, the back cover can maintain the integrity and beauty of the original appearance of the handheld terminal. The handheld NB-IoT wireless communication device provides the ability to collect NB-IoT signals and functions as a mobile phone case [27]. The NBIoT antenna is set close to the NB-IoT communication module, which improves the accuracy of signal reception. The back cover is provided with a through-hole, such as a camera through-hole and a headphone through-hole, directly facing the position of the camera or key on the handheld terminal, so that the handheld terminal can still use the functions of the handheld terminal after the handheld NB-IoT wireless communication [28] equipment is installed on the handheld terminal.

\section{Structure and Big Data Evaluation}

5.1. Structure Decomposition. The portable NB-IoT wireless communication device is fundamentally composed of a $\mathrm{CPU}$, an input device, an output device, and a memory. The schematic diagram of the handheld NB-IoT wireless communication device is shown in Figure 4. As illustrated in Figure 4, the memory is utilized to store a computer program. The computer program comprises program instructions, and the processor is set to invoke program instructions. The CPU 103, input devices, output devices, and memory are connected through buses 104, 102, 101, and 105. The memory is utilized to store 104 computer programs, which comprise instructions. The 103 processor is set to execute the instructions indicated in the program. The client communication driver module accomplishes the client and NB-IoT of communication between the communication modules by performing the following techniques. To realize network data transfer, the client TCP/IP protocol stack module receives and processes data and maps it to the handheld terminal, which can detect the network card device.

5.2. NB-IoT Big Data Processing. The IoT Big Data Processing function specifies processing the stored NB-IoT data for analysis and artificial intelligence application [29-33]. The IoT Big Data Processing feature also contains the Developer API Access Management. It provides authentication, authorization, and access control to govern access to intelligence and analysis to ensure privacy and security. We formulate and solve the model of the proposed application in this section. We begin by employing a 2dimensional Gaussian distribution to describe the number of NB-IoT and wireless internet sessions in the system. We further show that our model gives an analytical solution in the balance probability condition. Finally, the metrics of interest will be derived. We would also want to point out that the following evaluation technique is extensible enough to any mobile phone IoT technology [34, 35] using radio resources equivalent to those utilized by LTE. Figure 5 shows the performance transmission success rate of the NB-IoT terminal.

From Figure 5, we can observe that the lithium battery capacity of the NB-IoT terminal is $4000 \mathrm{mAh}$. When you evaluate the power consumption of the NB-IoT terminal, it is assumed that the terminal's microcontroller is awake once per day to collect and upload its battery and position information. After a significant amount of tests and measurements, we have found that terminal use is approximately $93 \mathrm{~mA}$. In comparison, the current operating on GPS module is at least $45 \mathrm{~mA}$, i.e., running microcontroller (larger while using an external antenna). Under the assumed working conditions, the terminal's battery life can only last up to 3.6 days if the switch triode is not utilized to manage the power supply of the GPS module. However, by employing a switch triode to manage the GPS module's power supply, the terminal's average total power consumption in the low-power state can be decreased to roughly $0.525 \mathrm{~mA}$ (i.e., the microcontroller is in the stop 


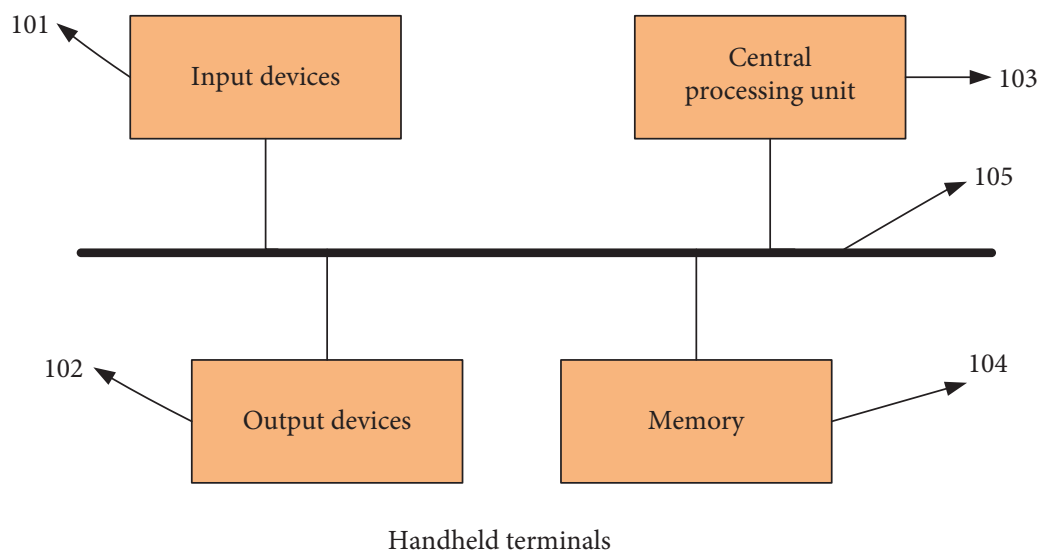

FIgURE 4: Schematic diagram of the handheld NB-IoT wireless communication device.

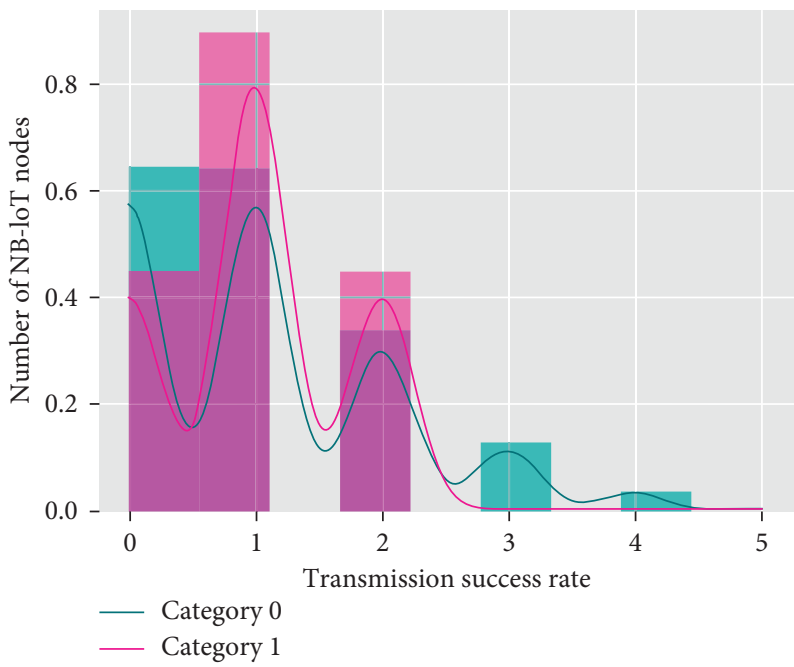

Figure 5: Performance transmission success rate of the NB-IoT wireless terminal.

mode). As a result, under the same estimated operating conditions, the terminal's battery life can reach several days.

\section{Conclusion}

NB-IoT is the most recent cellular technology, offering various configurations enabling massive IoT radio access from heterogeneous device groups. The combination of NBIoT portable terminals has replaced the previous $\mathrm{PC}+\mathrm{ATU}$, making tester testing easier. The test length, mobility, small size, and low power consumption are all raised effectively. In conjunction with today's standard mobile phone, tablet, and so on, the NB-IoT network optimization system has many benefits, including stability, portable operations, ease of use, and interactivity. Similarly, it is vital to examine the framework's design for handling extensive settings, optimum semistructured data processing, node scalability, and fault-tolerant storage structure. The solution presented would assist the engineer to imitate genuine methods of user perception testing to provide an efficient network optimization test to assist operators to grasp the quality and issues of the network more effectively.

\section{Data Availability}

The data used to support the findings of this study are included within the article.

\section{Conflicts of Interest}

The author declares that they have no conflicts of interest.

\section{References}

[1] M. Condoluci, M. A. Lema, T. Mahmoodi, and M. Dohler, “5g IoT industry verticals and network requirements," in Powering the Internet of Things With 5G Networks, pp. 148-175, IGI Global, Hershey, PA, USA, 2018.

[2] J. Seo, K. Kim, M. Park, M. Park, and K. Lee, "An analysis of economic impact on IoT industry under GDPR," Mobile Information Systems, vol. 20186 pages, Article ID 6792028, 2018.

[3] S.-H. Park and J.-K. Park, "IoT industry \& security technology trends," International Journal of Advanced Smart Convergence, vol. 5, no. 3, pp. 27-31, 2016.

[4] F. Tao, Y. Zuo, L. Da Xu, and L. Zhang, "IoT-based intelligent perception and access of manufacturing resource toward 
cloud manufacturing," IEEE Transactions on Industrial Informatics, vol. 10, no. 2, pp. 1547-1557, 2014.

[5] J. Wan, B. Chen, M. Imran et al., "Toward dynamic resources management for IoT-based manufacturing," IEEE Communications Magazine, vol. 56, no. 2, pp. 52-59, 2018.

[6] J. Cheng, W. Chen, F. Tao, and C.-L. Lin, "Industrial IoT in 5G environment towards smart manufacturing," Journal of Industrial Information Integration, vol. 10, pp. 10-19, 2018.

[7] M. Humayun, N. Jhanjhi, B. Hamid, and G. Ahmed, "Emerging smart logistics and transportation using IoT and blockchain," IEEE Internet of Things Magazine, vol. 3, no. 2, pp. 58-62, 2020.

[8] S. Selvaraj and S. Sundaravaradhan, "Challenges and opportunities in IoT healthcare systems: a systematic review," SN Applied Sciences, vol. 2, no. 1, pp. 1-8, 2020.

[9] D. Ugrenovic and G. Gardasevic, "CoAP protocol for Webbased monitoring in IoT healthcare applications," in Proceedings of the 2015 23rd Telecommunications Forum Telfor (TELFOR), pp. 79-82, IEEE, Belgrade, Serbia, November 2015.

[10] J. Yun, I.-Y. Ahn, N.-M. Sung, and J. Kim, “A device software platform for consumer electronics based on the internet of things," IEEE Transactions on Consumer Electronics, vol. 61, no. 4, pp. 564-571, 2015.

[11] M. S. Balaji and S. K. Roy, "Value co-creation with internet of things technology in the retail industry," Journal of Marketing Management, vol. 33, no. 1-2, pp. 7-31, 2017.

[12] E. Constantinides, M. Kahlert, and S. A. de Vries, "The relevance of technological autonomy in the acceptance of IoT services in retail," in Proceedings of the 2nd International Conference on Internet of Things, Data and Cloud Computing, ICC 2017, Cambridge, UK, March 2017.

[13] J. Chen, C. Du, Y. Zhang, P. Han, and W. Wei, "A clusteringbased coverage path planning method for autonomous heterogeneous UAVs," IEEE Transactions on Intelligent Transportation Systems, vol. 2021, Article ID 3066240, 11 pages, 2021.

[14] L. D. Xu, W. He, and S. Li, "Internet of things in industries: a survey," IEEE Transactions on Industrial Informatics, vol. 10, no. 4, pp. 2233-2243, 2014.

[15] Y. Huang, Y. Li, Z. Zhang, and R. W. Liu, "GPU-accelerated compression and visualization of large-scale vessel trajectories in maritime IoT industries," IEEE Internet of Things Journal, vol. 7, no. 11, pp. 10794-10812, 2020.

[16] Y. Xu, F. Jiang, J. Du, and D. Gong, "A cross-domain collaborative filtering algorithm with expanding user and item features via the latent factor space of auxiliary domains," Pattern Recognition, vol. 94, pp. 96-109, 2019.

[17] Y. Xu, Y. Chu, F. Jiang, Y. Guo, and D. Gong, "SVMs classification based two-side cross domain collaborative filtering by inferring intrinsic user and item features," KnowledgeBased Systems, vol. 141, pp. 80-91, 2018.

[18] Y. Xu, J. Yang, and Z. Xie, "Training SVMs on a bound vectors set based on fisher projection," Frontiers of Computer Science, vol. 8, no. 5, pp. 793-806, 2014.

[19] X. Yu, D. Zhan, L. Liu, H. Lv, L. Xu, and J. Du, "A privacypreserving cross-domain healthcare wearables recommendation algorithm based on domain-dependent and domainindependent feature fusion," IEEE Journal of Biomedical and Health Informatics, 2021, In press.

[20] M. Yu, T. Quan, Q. Peng, X. Yu, and L. Liu, "A model-based collaborate filtering algorithm based on stacked autoencoder," Neural Computing and Applications, 2021, In press.
[21] D. Turo, "Hierarchical visualization with treemaps: making sense of pro basketball data," in The Craft of Information Visualization, pp. 237-238, Morgan Kaufmann, Burlington, MA, USA, 2003.

[22] D. Miljković, L. Gajić, A. Kovačević, and Z. Konjović, "The use of data mining for basketball matches outcomes prediction," in Proceedings of the IEEE 8th International Symposium on Intelligent Systems and Informatics, IEEE, Subotica, Serbia, pp. 309-312, September 2010.

[23] B. J. Reich, J. S. Hodges, B. P. Carlin, and A. M. Reich, "A spatial analysis of basketball shot chart data," The American Statistician, vol. 60, no. 1, pp. 3-12, 2006.

[24] L. Guidetti, E. Franciosi, G. P. Emerenziani, M. C. Gallotta, and C. Baldari, "Assessing basketball ability in players with mental retardation," British Journal of Sports Medicine, vol. 43, no. 3, pp. 208-212, 2009.

[25] S. C. M. Te Wierike, M. C. De Jong, E. J. Y. Tromp et al., "Development of repeated sprint ability in talented youth basketball players," Journal of Strength and Conditioning Research, vol. 28, no. 4, pp. 928-934, 2014.

[26] N. Halevy, E. Y. Chou, A. D. Galinsky, and J. K. Murnighan, "When hierarchy wins: evidence from the national basketball association," Social Psychological and Personality Science, vol. 3, no. 4, pp. 398-406, 2012.

[27] G.-P. Zhou, D. Chen, S. Liao, and R.-B. Huang, "Recent progress in studying helix-helix interactions in proteins by incorporating the wenxiang diagram into the NMR spectroscopy," Current Topics in Medicinal Chemistry, vol. 16, no. 6, pp. 581-590, 2015.

[28] Q. Liu, L. Cheng, A. L. Jia, and C. Liu, "Deep reinforcement learning for communication flow control in wireless mesh networks," IEEE Network, vol. 35, no. 2, pp. 112-119, 2021.

[29] J. Zhang, Y. Liu, H. Liu, and J. Wang, "Learning local-global multiple correlation filters for robust visual tracking with kalman filter redetection," Sensors, vol. 21, no. 4, p. 1129, 2021.

[30] Y. Gu, A. Chen, X. Zhang, C. Fan, K. Li, and J. Shen, "Deep learning based cell classification in imaging flow cytometer," ASP Transactions on Pattern Recognition and Intelligent Systems, vol. 1, no. 2, pp. 18-27, 2021.

[31] J. Zhang, W. Wang, C. Lu, J. Wang, and A. K. Sangaiah, "Lightweight deep network for traffic sign classification," Annals of Telecommunications, vol. 75, no. 7, pp. 369-379, 2020.

[32] W. Sun, P. Zhang, Z. Wang, and D. Li, "Prediction of cardiovascular diseases based on machine learning," ASP Transactions on Internet of Things, vol. 1, no. 1, pp. 30-35, 2021.

[33] J. Zhang, X. Jin, J. Sun, J. Wang, and K. Li, "Dual model learning combined with multiple feature selection for accurate visual tracking," IEEE Access, vol. 7, pp. 43956-43969, 2019.

[34] G. Li and P. Zhang, "Design and implementation on patchouli growth environment monitoring system based on IoT," ASP Transactions on Internet of Things, vol. 1, no. 1, pp. 14-18, 2021.

[35] L. Yu, S. Tao, W. Gao, and L. Yu, "Self-monitoring method for improving health-related quality of life: data acquisition, monitoring, and analysis of vital signs and diet," ASP Transactions on Pattern Recognition and Intelligent Systems, vol. 1, no. 1, pp. 24-31, 2021. 